\title{
Modulational instability of a Langmuir wave in plasmas with energetic tails of superthermal electrons
}

\author{
I.V.Timofeev \\ Budker Institute of Nuclear Physics SB RAS, 630090, Novosibirsk, Russia \\ Novosibirsk State University, 630090, Novosibirsk, Russia
}

\begin{abstract}
The impact of superthermal electrons on dispersion properties of isotropic plasmas and on the modulational instability of a monochromatic Langmuir wave is studied for the case when the powerlaw tail of the electron distribution function extends to relativistic velocities and contains most of the plasma kinetic energy. Such an energetic tail of electrons is shown to increase the thermal correction to the Langmuir wave frequency, which is equivalent to the increase of the effective electron temperature in the fluid approach, and has almost no impact on the dispersion of ion-acoustic waves, in which the role of temperature is played by the thermal spread of low-energy core electrons. It is also found that the spectrum of modulational instability in the non-maxwellian plasma narrows significantly, as compared to the equilibrium case, without change of the maximum growth rate and the corresponding wavenumber.

PACS numbers: 52.35.Fp, 52.35.Mw, 52.35.-g
\end{abstract}

\section{INTRODUCTION}

The typical feature of turbulence evolution in a beamplasma system is the formation of superthermal tails on the distribution function of plasma electrons. These tails are often observed in space and laboratory plasmas. In particular, the electron distribution in the solar wind (see the review paper [1] and references therein) was found to be well approximated by the family of kappa-functions 2 26, decaying in velocity as a power law. Moreover, it has been shown recently that these functions describe asymptotically stationary solutions of weak turbulence equations, in which self-consistent evolution of both wave and particles spectra is taken into account $7-4$. In laboratory experiments, beam-plasma interaction appears to be much more intense than in the solar wind, that is why it results in the formation of strongly non-maxwellian electron distribution, in which most of the plasma kinetic energy is concentrated in a rather small population of fast electrons. Such a slowly decaying momentum distribution of plasma electrons $\left(f \propto p^{-5}\right)$ was really observed in the experiments on turbulent plasma heating by the powerful electron beam in the multi-mirror trap GOL-3 [10.

It is obvious that such an energetic tail of superthermal electrons should modify not only the linear dispersion of plasma modes, but also probabilities of various nonlinear processes responsible for formation of the turbulent spectrum. In the experiments of interest 11, the high-current relativistic electron beam interacts with the plasma in the regime of strong plasma turbulence. In this case, the main nonlinear process responsible for transferring the wave energy of beam-driven plasma oscillations to the nonresonant part of turbulent spectrum is the modulational instability. The goal of this paper is to investigate how the dispersion of linear plasma modes and the growth rate of the modulational instability pumped by the monochromatic Langmuir wave are modified in a strongly non-maxwellian plasma. Our interest to this problem is motivated by the need to interpret the experimental information about the intensity and the frequency spectrum of electromagnetic radiation generated in the turbulent plasma near the second harmonic of plasma frequency. Recent calculations of the radiation power [12, 13] for these experiments were based on the well known analytical model of strong plasma turbulence 14 . According to this model, the typical wavenumber of modulational instability determines the size of the energycontaining region of turbulent spectrum, and the typical growth rate allows to estimate the saturation level of turbulence energy. Thus, the study of the modulational instability in a non-maxwellian plasma will clarify whether superthermal electrons are able to modify the main parameters of emitting part of the turbulent spectrum.

The linear analysis of Langmuir waves in an isotropic plasma with a power-law momentum distribution in the framework of relativistic kinetic theory has been applied recently to the solar wind plasma near the orbit of the Earth [15]. In our paper, we study the dispersion properties of much hotter plasma that is typical to the laboratory beam-plasma experiments, and we focus on the validity of various widely used approximations. Investigations of modulational instability in relativistic plasmas with the Maxwell-Jüttner distribution [16, 17] or in the kappa-distributed plasmas with nonrelativistic superthermal tails [18, 19] are also of interest in last years. In contrast to these papers, we consider the modulational instability of a Langmuir wave in an unmagnetized plasma with the relativistic power-law momentum distribution of plasma electrons.

In Section II we obtain numerical solutions of the linear dispersion equation for the potential plasma waves of isotropic non-maxwellian plasma and compare them to the results predicted by fluid and kinetic approximations. In Section III, for the modulational instability, we deduce the dispersion equation that takes into account relativistic and kinetic effects for high-frequency 
oscillations, and compare numerical solutions for unstable spectra with the case of maxwellian plasma. In the concluding Section IV, we formulate our main results.

\section{LINEAR DISPERSION OF POTENTIAL PLASMA OSCILLATIONS}

Let us analyze the proper waves of non-maxwellian plasma assuming cold ions and using the following distribution function for electrons:

$$
f(\mathbf{p})=\frac{C_{0}}{4 \pi} \frac{H\left(p_{h}-p\right)}{\left(p^{2}+\Delta p^{2}\right)^{5 / 2}},
$$

where $H\left(p_{h}-p\right)$ is the Heaviside step function, $p_{h}$ is the threshold momentum, above which there are no electrons in the plasma, $\Delta p$ is the typical momentum spread of plasma electrons, and $C_{0}=3 \Delta p^{2} / \sin ^{3}\left(p_{h} / \Delta p\right)$ is the coefficient corresponding to the normalization $\int f d \mathbf{p}=1$. For the parameters $\Delta p=0.066 m_{e} c$ and $p_{h}=5 m_{e} c\left(m_{e}\right.$ is the rest mass of electons, $c$ is the speed of light), this function is best suited for the description of real electron distribution observed in beam-plasma experiments at the GOL-3 facility. The feature of this distribution function is that, for small momenta $p<\tilde{p}=2 \Delta p$, it does not differ greatly from the maxwellian function with the temperature $T=1 \mathrm{keV}$ and, for large momenta $p>\tilde{p}$, it has a power-law tail decreasing so slowly that most of the plasma kinetic energy is concentrated in superthermal electrons (fig. 1). The tail component of this particular

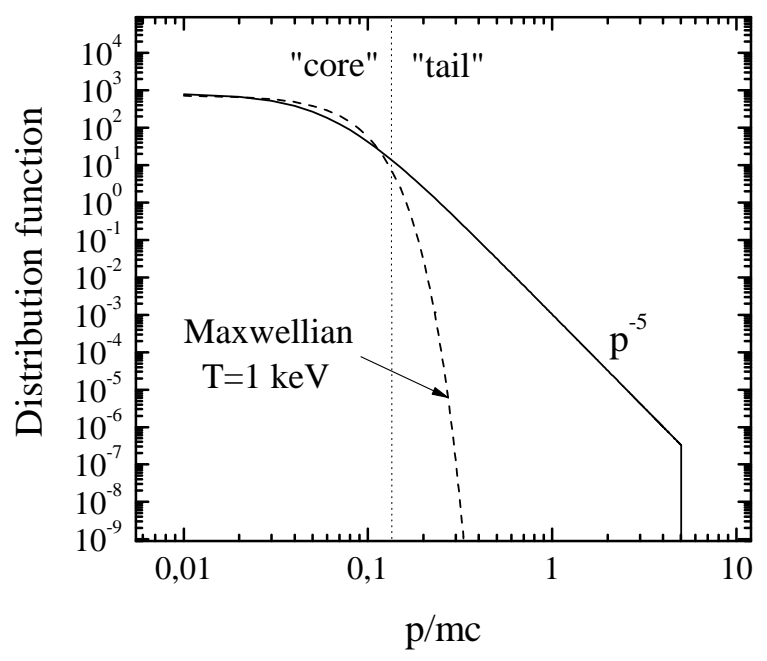

Figure 1: Momentum distribution of plasma electrons $f(\mathbf{p})$ for parameters typical to beam-plasma experiments at the GOL-3 facility.

distribution $(p>\tilde{p})$ contains $28 \%$ of the particles and $89 \%$ of the kinetic energy; the effective temperature of the whole distribution is

$$
T_{\text {eff }}=\frac{2}{3} \int m_{e} c^{2}(\gamma-1) f d \mathbf{p}=6.5 \mathrm{keV},
$$

whereas the temperature of core electrons with the relative density $n_{c}=72 \%$ turns out to be much smaller:

$$
T_{c}=\frac{2}{3 n_{c}} \int_{p<\tilde{p}} m_{e} c^{2}(\gamma-1) f d \mathbf{p}=0.97 \mathrm{keV}
$$

( $\gamma$ is the relativistic factor).

It is well known that dispersion laws of Langmuir and ion-acoustic waves in the maxwellian plasma have the following dimensionless forms:

$$
\begin{gathered}
\omega_{\ell} \simeq 1+\frac{3}{2} k^{2} T \\
\omega_{s} \simeq \sqrt{\frac{m_{e}}{m_{i}}} \frac{k T^{1 / 2}}{\sqrt{1+k^{2} T}} .
\end{gathered}
$$

Here frequencies are expressed in units of the plasma frequency $\omega_{p}=\left(4 \pi e^{2} n / m_{e}\right)^{1 / 2}$, where $e$ is the electron charge and $n$ is the plasma density, wavenumbers are measured in units of $\omega_{p} / c$, and the electron temperature in units of $m_{e} c^{2}$. These formulas have a simple hydrodynamic interpretation, according to which the electron pressure gradient $\nabla \delta p=\gamma_{e} T \nabla \delta n$ drives low-frequency acoustic oscillations of ions $\left(\gamma_{e}=1\right)$ and creates an additional force for high-frequency Langmuir oscillations of electrons $\left(\gamma_{e}=3\right)$. To adapt these insights to the case of non-maxwellian plasma, we should clarify what temperature $\left(T_{e f f}\right.$ or $\left.T_{c}\right)$ is more appropriate for the correct description of wave dispersion.

As we shall show later, the same expressions for real parts of wave frequencies can be derived from the exact kinetic theory, if we use the hydrodynamic approximation for Langmuir waves and kinetic approximation for ion-acoustic waves. From the nonrelativistic theory one can conclude that, for Langmuir oscillations, the role of temperature is played by

$$
T_{\ell}=\frac{1}{3} \int v^{2} f d \mathbf{p}=\left\langle\frac{v^{2}}{3}\right\rangle
$$

whereas, for ion-acoustic oscillations, the temperature is defined in another way

$$
T_{s}=\frac{1}{<1 / v^{2}>}
$$

In the maxwellian plasma with the temperature $T$, these values are equal, $T_{\ell}=T_{s}=T$, but even from these simple formulas one can see that relative contributions of superthermal electrons to $T_{\ell}$ and $T_{s}$ differ substantially. In a non-maxwellian plasma, the energetic tail component should lead to the significant increase of $T_{\ell}$, since it is proportional to the averaged kinetic energy of 
particles, and should not contribute to the value $T_{s}$, in which the inverse energy should be averaged. Thus, in the nonrelativistic case, superthermal electrons increase the thermal correction to the Langmuir wave frequency that is determined by the effective temperature of the whole distribution $T_{\text {eff }}$ and have almost no impact on ion-acoustic waves, for which the thermal spread of core electrons $T_{c}$ is more suitable to the role of temperature.

Let us now find out how these results change, if the tail of superthermal electrons extends to relativistic energies. First we will derive dispersion laws $\omega_{r}(k)$ and damping rates $\Gamma(k)$ of potential plasma waves in the hydrodynamic and kinetic limits, and then investigate applicability of these approximations by comparing approximate results with numerical solutions of the exact dispersion relation.

For slowly damping waves of isotropic plasma, the dispersion equation

$$
\varepsilon_{\|}=1+\frac{1}{k^{2}} \int \frac{\mathbf{k} \cdot \partial f / \partial \mathbf{p}}{\omega-\mathbf{k v}} d \mathbf{p}-\frac{m_{e} / m_{i}}{\omega^{2}}=0
$$

can be reduced to the form

$$
\operatorname{Re} \varepsilon_{\|}\left(\omega_{r}-i \Gamma, k\right)+i \operatorname{Im} \varepsilon_{\|}\left(\omega_{r}, k\right)=0,
$$

where real and imaginary parts of dielectric permittivity are defined by the integrals

$$
\begin{gathered}
\operatorname{Re} \varepsilon_{\|}=1+\frac{1}{k^{2}} V P \int \frac{\mathbf{k v}}{v} \frac{\partial f / \partial p}{\omega-\mathbf{k} \mathbf{v}} d \mathbf{p}-\frac{m_{e} / m_{i}}{\omega^{2}}, \\
\operatorname{Im} \varepsilon_{\|}=-\frac{\pi}{k^{2}} \int \frac{\mathbf{k v}}{v} \frac{\partial f}{\partial p} \delta(\omega-\mathbf{k v}) d \mathbf{p} .
\end{gathered}
$$

The dispersion law in this case is found from the equation

$$
\operatorname{Re} \varepsilon_{\|}\left(\omega_{r}, k\right)=0
$$

and the damping rate is calculated as

$$
\Gamma=\left(\frac{\operatorname{Im} \varepsilon_{\|}}{\partial \operatorname{Re} \varepsilon_{\|} / \partial \omega}\right)_{\omega=\omega_{r}} .
$$

We can calculate the electron contribution to $\operatorname{Re} \varepsilon_{\|}$using the hydrodynamic approximation $(\omega \gg \mathbf{k v})$ for Langmuir waves and the kinetic approximation $(\omega \ll \mathbf{k v})$ for ion-acoustic waves. It means that, in the former case, we can use the expansion

$$
\frac{1}{\omega-\mathbf{k v}}=\frac{1}{\omega}\left(1+\frac{\mathbf{k v}}{\omega}+\frac{(\mathbf{k v})^{2}}{\omega^{2}}+\frac{(\mathbf{k v})^{3}}{\omega^{3}} \ldots\right)
$$

and in the latter case

$$
\frac{1}{\omega-\mathbf{k v}}=-\frac{1}{\mathbf{k v}}+\ldots
$$

$\operatorname{Im} \varepsilon_{\|}$is determined for both types of waves by the unified expression:

$$
\operatorname{Im} \varepsilon_{\|}=2 \pi^{2} \frac{\omega}{k^{3}}\left[\gamma_{0}^{2} f\left(p_{0}\right)+2 \int_{p_{0}}^{\infty} p f d p\right],
$$

where $p_{0}$ is the momentum of electrons getting into Cherenkov resonance with the wave,

$$
p_{0}=\frac{\omega_{r} / k}{\sqrt{1-\omega_{r}^{2} / k^{2}}}
$$

and $\gamma_{0}=\sqrt{1+p_{0}^{2}}$ is the corresponding relativistic factor. Thus, the real frequency of Langmuir wave is governed by the equation

$$
1-\frac{\omega_{0}^{2}}{\omega_{r}^{2}}-3 \frac{k^{2} T_{\ell}}{\omega_{r}^{4}}=0
$$

where

$$
\begin{gathered}
\omega_{0}^{2}=\int \frac{f}{\gamma}\left(1-\frac{v^{2}}{3}\right) d \mathbf{p}=\left\langle\frac{1-v^{2} / 3}{\gamma}\right\rangle, \\
T_{\ell}=\left\langle\frac{v^{2}}{3 \gamma}\left(1-\frac{3}{5} v^{2}\right)\right\rangle,
\end{gathered}
$$

and the real frequency of ion-acoustic wave is determined by

$$
1+\frac{1}{k^{2} T_{s}}-\frac{m_{e} / m_{i}}{\omega_{r}^{2}}=0
$$

where

$$
T_{s}^{-1}=\left\langle\frac{1+v^{2}}{p v}\right\rangle .
$$

In the nonrelativistic limit, $\omega_{0}^{2} \rightarrow 1$ and calculation of electron temperature really reduces to averaging either the kinetic energy $T_{\ell} \rightarrow<v^{2} / 3>$ or the reciprocal value $T_{s}^{-1} \rightarrow<1 / v^{2}>$. From these formulas, it is seen that relativistic effects manifest themselves in decreasing the plasma frequency $\left(\omega_{0}^{2}<1\right)$ due to weighting of tail electrons. Moreover, the temperature $T_{\ell}$ in the relativistic case ceases to be proportional to the total kinetic energy and can no longer be identified with the value $T_{\text {eff }}=<2(\gamma-1) / 3>$. Indeed, the expression averaged in $T_{\ell} 18$ differs from the value $2(\gamma-1) / 3$ by the factor

$$
F=\frac{(\gamma+1)}{5 \gamma^{3}}\left(1+\frac{3}{2 \gamma^{2}}\right)
$$

which substantially reduces the relative contribution of fast electrons to the thermal correction to the Langmuir wave frequency. As to the temperature $T_{s}$, its calculation for both low $\gamma \ll 1$ and high $\gamma \gg 1$ energies reduces to averaging the value that is inversely proportional to the energy. It means that relativistic effects do not change the earlier conclusion that superthermal electrons do not contribute to the electron pressure perturbation driving lowfrequency acoustic oscillations. For the distribution function (1), the temperature $T_{\ell}$ accounting for the relativistic effects appears to be twice smaller than the effective temperature of the whole distribution $\left(T_{\ell}=0.56 T_{\text {eff }}\right)$ 
and the temperature $T_{s}$ remains close to the temperature of low-energy core electrons $\left(T_{s}=1.15 T_{c}\right)$ as in the nonrelativistic case.

The exact dispersion relation for the damping plasma modes can be written in the form

$$
\begin{aligned}
& \varepsilon_{\|}(\omega, k)=1-\frac{m_{e} / m_{i}}{\omega^{2}}+\frac{4 \pi}{k^{2}} \int_{0}^{\infty} \frac{f}{\gamma}\left(\gamma^{2}+p^{2}\right) d p \\
& -2 \pi \frac{\omega}{k^{3}} \int_{0}^{\infty} \gamma^{2} \frac{\partial f}{\partial p} \ln \left(\frac{\omega-k v}{\omega+k v}\right) d p+i \operatorname{Im} \varepsilon_{\|}=0
\end{aligned}
$$

where the imaginary part of dielectric permittivity is still defined by 15 and the function $\ln (\ldots)$ means the principal branch of complex logarithm. For Langmuir waves, the comparison of numerical solution of this equation $\omega=\omega_{r}-i \Gamma$ with the results of the hydrodynamic approximation

$$
\begin{gathered}
\omega_{r}=\left(\frac{\omega_{0}^{2}+\sqrt{\omega_{0}^{4}+12 k^{2} T_{\ell}}}{2}\right)^{1 / 2}, \\
\Gamma=\frac{\pi \omega_{r}^{4} C_{0}}{6 k^{3}\left(\omega_{0}^{2}+6 k^{2} T_{\ell} / \omega_{r}^{2}\right)\left(p_{0}^{2}+\Delta p^{2}\right)^{5 / 2}} \times \\
{\left[\frac{3 \gamma_{0}^{2}}{2}+p_{0}^{2}+\Delta p^{2}-\frac{\left(p_{0}^{2}+\Delta p^{2}\right)^{5 / 2}}{\left(p_{h}^{2}+\Delta p^{2}\right)^{3 / 2}}\right],}
\end{gathered}
$$

is shown in Fig. 2, It is seen that the approximate so-
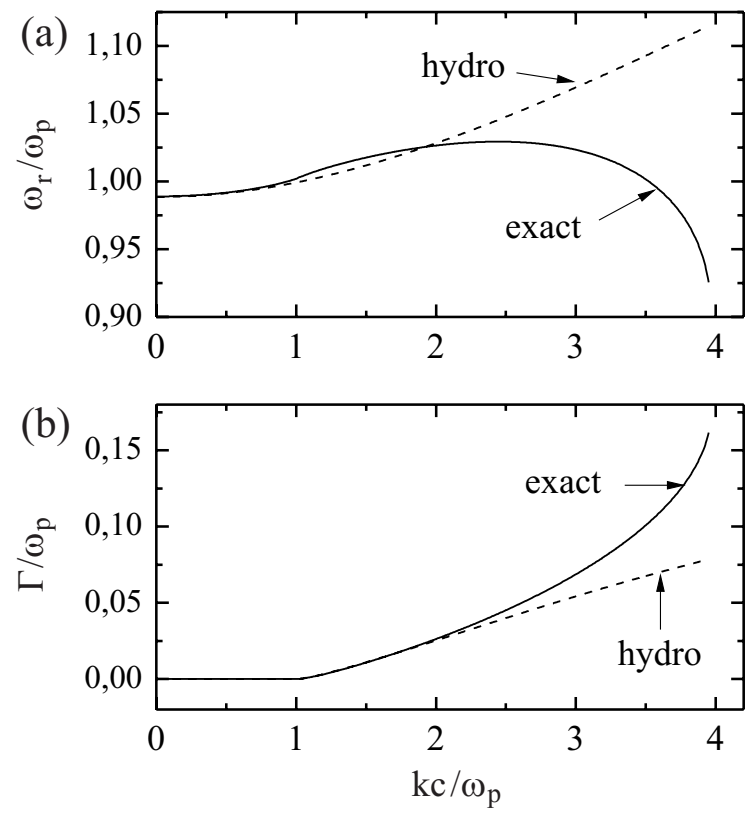

Figure 2: The dispersion (a) and Landau damping (b) of Langmuir waves in the non-maxwellian plasma.

lution for the parameters of interest is adequate in the long-wavelength $(k<2)$ part of the spectrum only. Oscillations with shorter wavelength falls in the region of strong Landau damping and demonstrate the anomalous dispersion $\left(\partial \omega_{r} / \partial k<0\right)$. For ion-acoustic waves, on the contrary, the kinetic approximation 19 turns out to be so accurate that both real and imaginary parts of complex frequency fit the numerical solution of exact dispersion equation in the wide range of wavenumbers (fig. 3.
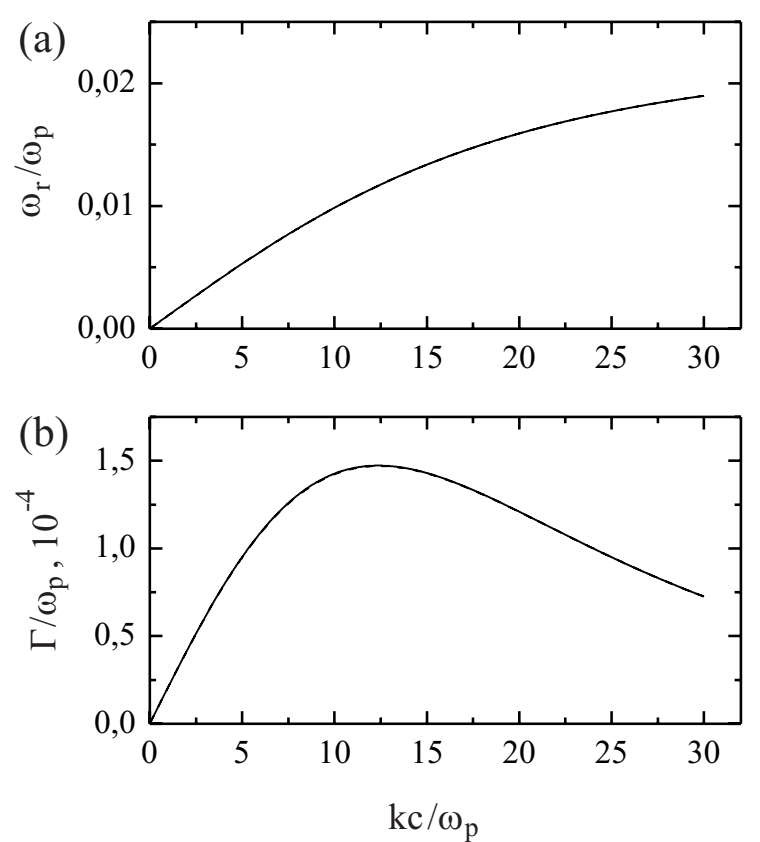

Figure 3: The dispersion (a) and Landau damping (b) of ionacoustic waves in the non-maxwellian plasma (numerical solutions of equation (22) coincide with the results of kinetic approximation).

\section{MODULATIONAL INSTABILITY}

Let us consider stability of the monochromatic Langmuir wave

$$
\mathbf{E}(t, \mathbf{r})=\frac{1}{2}\left(\mathbf{E}_{\mathbf{0}} e^{-i \omega_{k} t+i \mathbf{k r}}+\text { c.c. }\right)
$$

with respect to the coupled system of low-frequency and high-frequency modulational perturbations

$$
\begin{aligned}
& \delta \mathbf{E}(t, \mathbf{r})=\frac{\delta \mathbf{E}}{2} e^{-i \Omega t+i \mathbf{q} \mathbf{r}}+\frac{\delta \mathbf{E}_{+}}{2} e^{-i \omega_{+} t+i \mathbf{k}_{+} \mathbf{r}} \\
& +\frac{\delta \mathbf{E}_{-}}{2} e^{-i \omega_{-} t+i \mathbf{k}_{-} \mathbf{r}}+c . c .
\end{aligned}
$$

where

$$
\omega_{ \pm}=\omega_{k} \pm \Omega, \quad \mathbf{k}_{ \pm}=\mathbf{k} \pm \mathbf{q}
$$


In the plasma with cold ions and arbitrarily distributed electrons, equations for the amplitudes of potential highfrequency satellites can be written in the following form:

$$
\begin{aligned}
& \varepsilon_{+} \delta E_{+}=\frac{e^{2} E_{0}}{4 k k_{+}} \times \\
& {\left[\frac{E_{0}^{*} \delta E_{+}}{k k_{+}}\left(\frac{G_{1}^{+} G_{4}}{q^{2} \varepsilon_{q}}-G_{2}^{+}\right)+\frac{E_{0} \delta E_{-}^{*}}{k k_{-}}\left(\frac{G_{1}^{+} G_{5}}{q^{2} \varepsilon_{q}}-G_{3}^{+}\right)\right]} \\
& \varepsilon_{-}^{*} \delta E_{-}^{*}=\frac{e^{2} E_{0}^{*}}{4 k k_{-}} \times \\
& {\left[\frac{E_{0}^{*} \delta E_{+}}{k k_{+}}\left(\frac{G_{1}^{-} G_{4}}{q^{2} \varepsilon_{q}}-G_{2}^{-}\right)+\frac{E_{0} \delta E_{-}^{*}}{k k_{-}}\left(\frac{G_{1}^{-} G_{5}}{q^{2} \varepsilon_{q}}-G_{3}^{-}\right)\right],}
\end{aligned}
$$

where

$$
\begin{aligned}
& G_{1}^{ \pm}=4 \pi e^{2} \int \frac{d^{3} p}{\omega_{ \pm}-\mathbf{k}_{ \pm} \mathbf{v}} \times \\
& {\left[\mathbf{k} \cdot \partial_{\mathbf{p}}\left(\frac{\mathbf{q} \cdot \partial_{\mathbf{p}} f}{\Omega-\mathbf{q} \mathbf{v}}\right) \pm \mathbf{q} \cdot \partial_{\mathbf{p}}\left(\frac{\mathbf{k} \cdot \partial_{\mathbf{p} f}}{\omega_{k}-\mathbf{k} \mathbf{v}}\right)\right],} \\
& G_{2}^{ \pm}=4 \pi e^{2} \int d^{3} p \frac{\mathbf{k} \cdot \partial_{\mathbf{p}}}{\omega_{ \pm}-\mathbf{k}_{ \pm} \mathbf{v}} \times \\
& {\left[\frac{\mathbf{k}_{+} \cdot \partial_{\mathbf{p}}}{\Omega-\mathbf{q} \mathbf{v}}\left(\frac{\mathbf{k} \cdot \partial_{\mathbf{p}} f}{\omega_{k}-\mathbf{k v}}\right)-\frac{\mathbf{k} \cdot \partial_{\mathbf{p}}}{\Omega-\mathbf{q} \mathbf{v}}\left(\frac{\mathbf{k}_{+} \cdot \partial_{\mathbf{p}} f}{\omega_{+}-\mathbf{k}_{+} \mathbf{v}}\right)\right],} \\
& G_{3}^{ \pm}=4 \pi e^{2} \int d^{3} p \frac{\mathbf{k} \cdot \partial_{\mathbf{p}}}{\omega_{ \pm}-\mathbf{k}_{ \pm} \mathbf{V}} \times \\
& {\left[\frac{\mathbf{k} \cdot \partial_{\mathbf{p}}}{\Omega-\mathbf{q} \mathbf{v}}\left(\frac{\mathbf{k}_{-} \cdot \partial_{\mathbf{p}} f}{\omega_{-}-\mathbf{k}_{-} \mathbf{v}}\right)-\frac{\mathbf{k}_{-} \cdot \partial_{\mathbf{p}}}{\Omega-\mathbf{q} \mathbf{v}}\left(\frac{\mathbf{k} \cdot \partial_{\mathbf{p}} f}{\omega_{k}-\mathbf{k} \mathbf{v}}\right)\right],} \\
& G_{4}=4 \pi e^{2} \int \frac{d^{3} p}{\Omega-\mathbf{q v}} \times \\
& {\left[\mathbf{k}_{+} \cdot \partial_{\mathbf{p}}\left(\frac{\mathbf{k} \cdot \partial_{\mathbf{p}} f}{\omega_{k}-\mathbf{k} \mathbf{v}}\right)-\mathbf{k} \cdot \partial_{\mathbf{p}}\left(\frac{\mathbf{k}_{+} \cdot \partial_{\mathbf{p}} f}{\omega_{+}-\mathbf{k}_{+} \mathbf{v}}\right)\right] \text {, }} \\
& G_{5}=4 \pi e^{2} \int \frac{d^{3} p}{\Omega-\mathbf{q} \mathbf{v}} \times \\
& {\left[\mathbf{k} \cdot \partial_{\mathbf{p}}\left(\frac{\mathbf{k}_{-} \cdot \partial_{\mathbf{p}} f}{\omega_{-}-\mathbf{k}_{-} \mathbf{v}}\right)-\mathbf{k}_{-} \cdot \partial_{\mathbf{p}}\left(\frac{\mathbf{k} \cdot \partial_{\mathbf{p}} f}{\omega_{k}-\mathbf{k v}}\right)\right],} \\
& \varepsilon_{ \pm}=\varepsilon_{\|}\left(\omega_{ \pm}, \mathbf{k}_{ \pm}\right), \quad \varepsilon_{q}=\varepsilon_{\|}(\Omega, \mathbf{q}) .
\end{aligned}
$$

In the limiting cases $\Omega \ll \mathbf{q v}$ and $\omega_{k, \pm} \gg \mathbf{k}_{ \pm} \mathbf{v}$, all integrals $G_{i}$ reduce to averaging $\left.<1 / v^{2}\right\rangle$. Thus, the main contribution to these integrals comes from low-energy core electrons, the typical velocity of which is really much smaller than phase velocities of high-frequency satellites and the speed of light. It allows us to neglect relativistic effects in calculations of $G_{i}$ and transform the equations to the well-known form 20 ]

$\varepsilon_{+} \delta E_{+}=-\frac{E_{0} \cos \theta_{+}}{16 \pi n T_{s}} \frac{\varepsilon_{q}^{i}}{\varepsilon_{q}}\left(E_{0}^{*} \delta E_{+} \cos \theta_{+}+E_{0} \delta E_{-}^{*} \cos \theta_{-}\right)$, $\varepsilon_{-}^{*} \delta E_{-}^{*}=-\frac{E_{0}^{*} \cos \theta_{-}}{16 \pi n T_{s}} \frac{\varepsilon_{q}^{i}}{\varepsilon_{q}}\left(E_{0}^{*} \delta E_{+} \cos \theta_{+}+E_{0} \delta E_{-}^{*} \cos \theta_{-}\right)$

$$
\varepsilon_{q}^{i}=-\omega_{p i}^{2} / \Omega^{2}, \quad \cos \theta_{ \pm}=\frac{\mathbf{k k}_{ \pm}}{k k_{ \pm}}
$$

The specific features of non-maxwellian plasma in these equations manifest themselves only in changing of temperature notation from $T$ to $T_{s}$ and in accurate calculation of permittivities $\varepsilon_{ \pm}$in the framework of relativistic kinetic theory.

Thus, the modulational instability in a non-maxwellian plasma can be described by the following equation:

$$
\Omega^{2}-\Omega_{s}^{2}=W \Omega_{s}^{2}\left[\frac{\cos ^{2} \theta_{+}}{\varepsilon_{+}}+\frac{\cos ^{2} \theta_{-}}{\varepsilon_{-}^{*}}\right]
$$

where

$$
\Omega_{s}^{2}=\frac{m_{e}}{m_{i}} \frac{q^{2} T_{s}}{1+q^{2} T_{s}}
$$

corresponds to the linear dispersion of ion-acoustic waves, and the parameter

$$
W=\frac{\left|E_{0}\right|^{2}}{16 \pi n T_{s}}
$$

determines the relative energy of the Langmuir pump wave. Since we look for unstable solutions of $(37) \Omega=$ $\operatorname{Re} \Omega+i \operatorname{Im} \Omega$ with $\operatorname{Im} \Omega>\operatorname{Im} \omega_{k}$, the last term in the definition of $\varepsilon_{\|}$, appearing as a result of analytical continuation of this function to the lower half-plane of $\omega$, must be omitted.

For example, let us find a numerical solution of the dispersion relation (37) in the case when the role of longwavelength pump is played by the undamped Langmuir wave with the wavenumber $k=1$, the frequency $\omega_{k}=$ 1.00203 and the energy $W=0.01$. Fig. 4 shows that the growth rate of modulational instability $\operatorname{Im} \Omega\left(q_{\perp}, q_{\|}\right)$ reaches the maximum for the perturbations propagating along $\mathbf{k}$. Since this maximum falls in the region of rather small wavenumbers $q_{\|} \simeq 2$, it is reasonable to estimate how accurately the unstable spectrum can be described by the fluid approach, in which thermal corrections to the dispersion of high-frequency oscillations depends on the increased effective temperature $T_{\ell}>T_{s}$. In this limit, the functions $\varepsilon_{ \pm}$in the dispersion equation should have the simple form

$$
\varepsilon_{ \pm}=1-\frac{1+3 k_{ \pm}^{2} T_{\ell}}{\omega_{ \pm}^{2}} .
$$

The unstable spectrum of longitudinally propagating modulational perturbations $\left(q_{\perp}=0\right)$ in comparison with the case of maxwellian plasma $\left(T_{\ell}=T_{s}\right)$ is shown in Fig. 5. It is seen that the increase in the temperature $T_{\ell}$ with respect to $T_{s}$ in the hydrodynamic limit results in almost the same narrowing of the spectrum of modulational instability (by the factor $T_{\ell} / T_{s}$ ) that is observed in the case 


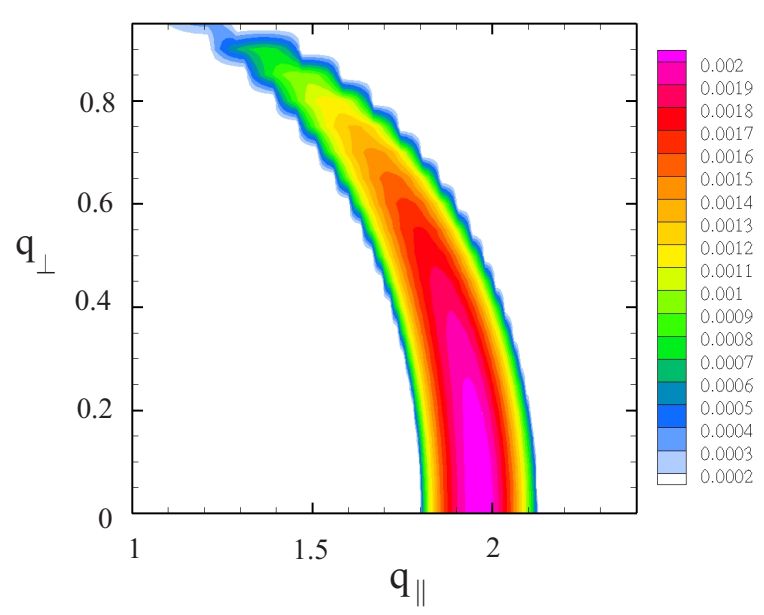

Figure 4: The growth-rate map for the modulational instability pumped by a monochromatic Langmuir wave.

of exact kinetic description of Langmuir waves. Another important result is that the appearance of the tail component in the electron distribution does not change neither the maximum of the growth rate nor its position in the wavenumber space. It means that we can estimate the typical gtowth rate and the corresponding wavenumber of modulational instability in the non-maxwellian plasma without the detailed study of the real distribution function. For this purpose, we can restrict ourselves by the case of maxwellian plasma with the temperature corresponding to the temperature of core electrons.

\section{SUMMARY}

In the present study we have calculated dispersion laws and damping rates of Langmuir and ion-acoustic oscillations in the case, when an isotropic plasma has the energetic tail of superthermal electrons typical to the beam-plasma experiments at the multi-mirror trap GOL3 . The specific feature of such distribution is that most of the kinetic energy is concentrated in a small population of fast electrons. The concept of temperature in this case loses its usual meaning, since the energy spread of the bulk of electrons appears to be much smaller than the total kinetic energy. Nevertheless, the dispersion of linear Langmuir waves in the long-wavelength region can be correctly described by the fluid approach, in which the effective temperature $T_{\ell}$ exceeds substantially the temperature of core electrons $T_{c}$, but, due to relativistic effects, does not reach the temperature of the whole distribution. Ion-acoustic waves, in turn, are shown to be well described by the kinetic approximation with the temperature of low-energy core electrons $T_{s} \approx T_{c}$.

Modifications in the linear dispersion of high-frequency waves in a non-maxwellian plasma should have an impact on the build-up efficiency of modulational insta-
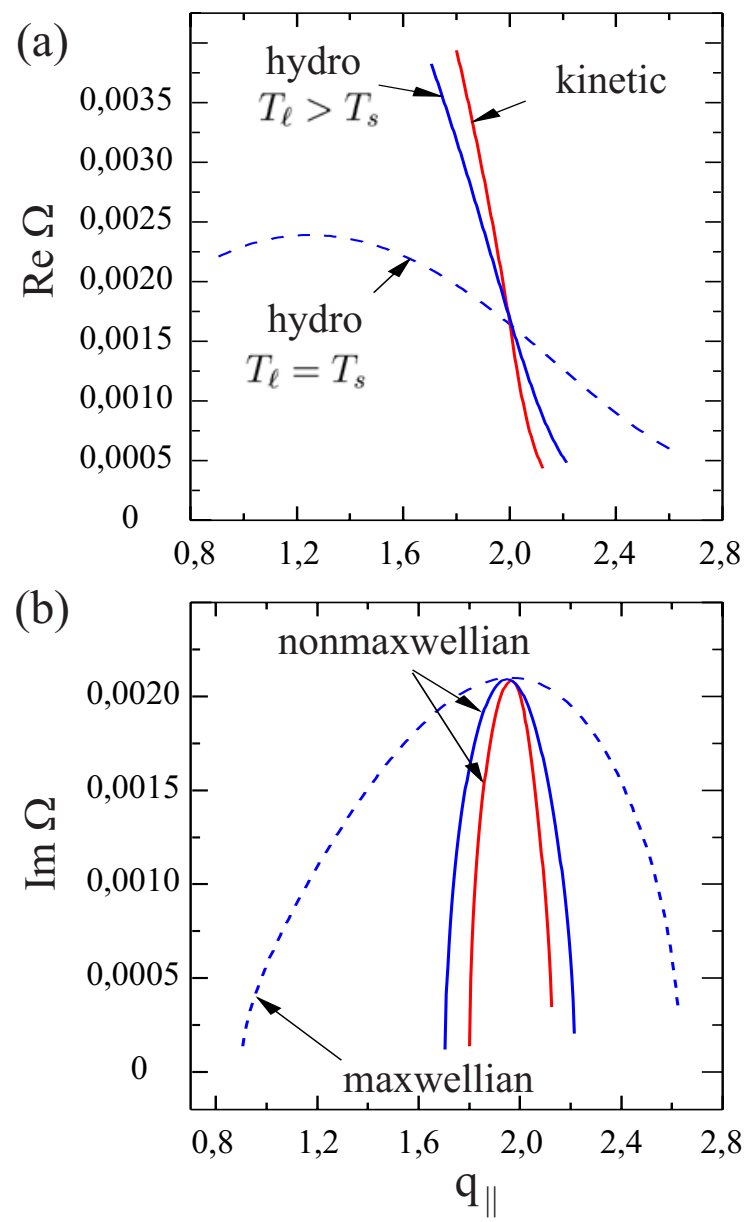

Figure 5: The real (a) and imaginary (b) parts of the frequency of longitudinally propagating modulational perturbations (the fluid approximation for the maxwellian plasma with $T_{\ell}=T_{s}$ - dashed line, the fluid approximation for the nonmaxwellian plasma with the increased temperature $T_{\ell}$ - blue solid line, numerical solution of (37) with the exact kinetic expressions for $\varepsilon_{ \pm}-$red solid line).

bility playing the important role in the scenarios of strong plasma turbulence. We have solved numerically the dispersion equation for modulational perturbations, in which both kinetic and relativistic effects are taken into account. It is shown that the unstable spectrum in the non-maxwellian plasma narrows significantly without changing in the growth rate and wavelength of the most unstable perturbation. It is also found that the same trend is qualitatively reproduced in the case when highfrequency waves are described by the fluid approximation with the increased effective temperature $T_{\ell}>T_{s}$.

Independence of the typical growth rate and the wavelength of modulational instability on distribution details of superthermal electrons allows to prove the possibility of applying the model of strong Langmuir turbulence [12], used for calculation of electromagnetic plasma emission, to the case of realistic non-maxwellian plasmas typical to 
laboratory beam-plasma experiments.

The author thanks K.V.Lotov for useful discussions. The study was supported by The Ministry of education and science of Russia (projects 14.B37.21.0750,
14.B37.21.1178 and 14.B37.21.0784), Russian Foundation of Basic Research (grants 12-02-31696, 11-02-00563), grant of RF Government 11.G34.31.0033 and President grants SP-1289.2012.1 and NSh-5118.2012.2.
[1] V. Pierrard, M. Lazar, Sol. Phys. 267, 153 (2010).

[2] V.M. Vasyliunas, J. Geophys. Res. 73, 2839, doi: 10.1029/ JA073i009p02839 (1968).

[3] D. Summers and R.M. Thorne, Phys. Fluids B B3, 1835 (1991).

[4] Y.F. Chateau and N. Meyer-Vernet, J. Geophys. Res. 96, 5825, DOI: 10.1029/90JA02565 (1991).

[5] R.L. Mace and M.A. Hellberg, Phys. Plasmas 2, 2098 (1995).

[6] M.A. Hellberg, R.L. Mace, T.K. Baluku, I. Kourakis, and N.S. Saini, Phys. Plasmas 16, 094701 (2009).

[7] P.H. Yoon, Phys. Plasmas 18, 122303 (2011).

[8] P.H. Yoon, Phys. Plasmas 19, 012304 (2012).

[9] P.H. Yoon, Phys. Plasmas 19, 052301 (2012).

[10] V.T. Astrelin, A.V. Burdakov, P.Z. Chebotaev, V.V. Filippov, V.S. Koidan, K.I. Mekler, P.I.Melnikov, V.V. Postupaev, A.F. Rovenskikh, M.A. Shcheglov, H. Wuerz, Nucl. Fusion 37, 1541 (1997).

[11] A.V. Arzhannikov, A.V. Burdakov, S.A. Kuznetsov,
M.A. Makarov, K.I. Mekler, V.V. Postupaev, A.F. Rovenskikh, S.L. Sinitsky, V.F. Sklyarov, Fusion Sci. and Technol. 59 (1T), 74 (2011).

[12] I.V. Timofeev, Phys. Plasmas 19, 044501 (2012).

[13] A.V. Arzhannikov and I.V. Timofeev, Plasma Phys. Control. Fusion 54, 105004 (2012).

[14] A.A. Galeev, R.Z. Sagdeev, V.D. Shapiro, and V.I. Shevchenko, Sov. Phys. JETP 46, 711 (1977).

[15] J.J. Podesta, Phys. Plasmas 15, 122902 (2008).

[16] X.L. Liu, X.Q. Li, and S.Q. Liu, Phys. Plasmas 18, 082301 (2011).

[17] X.L. Liu, S.Q. Liu, X.Q. Li, Phys. Plasmas 19, 092101 (2012).

[18] S.Q. Liu, H. Chen, Phys. Plasmas 19, 012303 (2012).

[19] L.A. Rios and M.O. Galvao, Phys. Plasmas 17, 042116 (2010).

[20] F.Kh. Khakimov and V.N. Tsytovich, Zh. Eksp. Teor. Fiz. 70, 1785 (1976). 